\title{
Die Wirkung vollständig abgebauter Nahrung auf den Verdauungskanal. \\ Von \\ Otto Cohnheim.
}

(Der Redaktion zugegangen am 23. März 1913.)

Eine Reihe von Stoffwechselversuchen ${ }^{1}$ ) der letzten Jahre haben ergeben, daß es gelingt, Tiere, auch Menschen, ${ }^{2}$ ) mit vollständig abgebauten, d. h. in Aminosäuren zerlegtem Eiweiß in Stickstoffgleichgewicht $\mathrm{zu}$ bringen. Bei diesen Versuchen hatte sich gezeigt, daß der Organismus imstande ist, die Aminosäuren wie das Eiweiß zu verwerten. Über die Art, wie die abgebaute Nabrung aber auf die Verdauungsorgane wirkt, gestattet die Versuchsanordnung der Stoffwechselversuche keinen Aufschluß. Nur das eine hat sich bei ihnen ergeben, daß das Gemenge der Aminosäuren im Gegensatz zu Peptonen keine Durchfälle hervorruft, und daß es auch bei der Eingabe per rectum gehalten und resorbiert. wird. ${ }^{2}$ ) Die Frage nach der Einwirkung der abgebauten Nahrung auf den Verdauungskanal ist aber von einem gewissen Interesse. Nach allem, was wir wissen, ist für den Ablauf der Verdauungsvorgänge neben dem Wohlgeschmack die chemische-Zusammensetzung der Nahrung von entscheidender Bedeutung. Aber auch die Konsistenz ist für die Bewegungen nicht bedeutungslos. Durch den Abbau der Nahrung wird die Konsistenz vollständig geändert, wie weit die chemische Zusammensetzung sich wirksam ändert,

1) E. Abderhalden, Diese Zeitschrift, Bd. 77, S. 22, 1912. Daselbst sind die früheren Arbeiten von Abderhalden und seinen Mitarbeitern zitiert. Vgl. auch 0 . Cohnheim, Physiol. d. Verdauung und Ernährung, S. 223, 1908.

2) E. Abderhalden, F. Frank u. A. Schittenhelm, Diese Zeitschrift, Bd. 63, S. 215, 1909.

Hoppe-Seyler's Zeitschrift f. physiol. Chemie. LXXXIV. 
ist die Frage. Weiterhin ist die Frage nach der Verdauung vollständig abgebauter Nahrung auch von einem gewissen praktischen Interesse, da neuerdings Nährpräparate aus vollständig abgebauter Nahrung in den Handel gebracht werden, die nach den Angaben der Firmen keine Eiweißpräparate sind, sondern aus den vollständigen Nahrungsmitteln ohne Verlust dargestellt werden. Gegen die früheren Eiweißpräparate mußte vom Standpunkte des Physiologen der schwerwiegende Einwand erhoben werden, daß sie eben nur Eiweißpräparate waren, und daß die anderen Stoffe, besonders die anderen stickstoffhaltigen Stoffe, in ihnen fehlten. ${ }^{1}$ ) Die große Bedeutung der verschiedenen chemischen Körper, die neben dem Eiweiß in den Nahrungsmitteln vorhanden sind, ist ja längst bekannt, sie ist aber neuerdings durch die Untersuchungen von Stepp in besonders schlagender Weise demonstriert worden. ${ }^{2}$ ) Stepp zeigte, daß die Extraktion von Eiweißkörpern mit Alkohol und Äther einen Stoff entfernt, dessen Fehlen die Nahrung unfähig macht, das Leben zu erhalten. Gelingt es, ganze Nahrungsmittel abzubauen und in Lösung zu bringen, so liegt die Sache wesentlich anders, derartige Nährpräparate können, abgesehen natürlich von den Fragen des Preises, die den Physiologen nichts angehén, vom physiologischen Standpunkte aus, als richtig bezeichnet werden. Ich habe daher an Hunde mit Duodenalfisteln vollständig abgebaute Nahrung verfüttert und nachgesehen, wie sich Sekretion und Motilität verhalter. Als abgebauter Nahrung bediente ich mich des Ereptons, das nach Abderhaldens Angaben von den Höchster Farbwerken in Handel gebracht wird, und des Hapans, das von Theinhardts Nährmittelgesellschaft in Handel gebracht und als vollständige und vollständig abgebaute Nahrung bezeichnet wird. Es wurde mir von der Firma in liebenswürdiger Weise zur Verfügung gestellt.

Hapan hat die Konsistenz, den Geruch und Geschmack von Liebigs Fleischextrakt. Es gibt die Biuretreaktion nicht,

1) 0. Cohnheim, l. c., S. 261, 467.

2) W. Stepp, Zeitschr. f. Biol., Bd. 57, S. 135 (1911); Bd. 59, S. 366 (1912). 
Wirkung vollständig abgebauter Nahrung auf den Verdauungskanal. 421

aber alle anderen Farbenreaktionen des Eiweiß, auch die von Tryptophan, sodaß bei der Darstellung offenbar keiner der Bausteine zerstört worden ist. In kaltem Wasser löst es sich langsam und unvollständig, in heißem Wasser löst es sich zum größten Teile sehr leicht, ein geringer Rückstand, der vorwiegend aus Tyrosin besteht, etwas schwerer. Ist es einmal gelöst, so bleibt es auch beim Abkühlen in Lösung. Es enthält etwa 10\% Stickstoff; von den Hunden wurde es gierig gesoffen. - Der Stickstoffgehalt des Ereptons beträgt nach dem Aufdruck 12,7\%.

Ich gebe ein vollständiges Versuchsprotokoll. Zum Versuche diente ein Hund, der eine Duodenalfistel hatte und bei dem außerdem eine Anastomose zwischen der Gallenblase und einer Dünndarmschlinge bestand, während der Ductus choledochus unterbunden war. Bei diesem Hunde entleerte sich also aus der Duodenalfistel keine Galle, sondern nur Magenund Pankreassaft. Es ist dasselbe Tier, das Klee und ich im vorigen Jahr beschrieben haben. ${ }^{1}$ ) Das Tier erhielt $20 \mathrm{~g}$ Hapan, in $250 \mathrm{ccm}$ Wasser gelöst, bei offener Duodenalfistel zu saufen; das, was sich aus der Fistel entleerte, wurde immer sofort wieder eingespritzt.

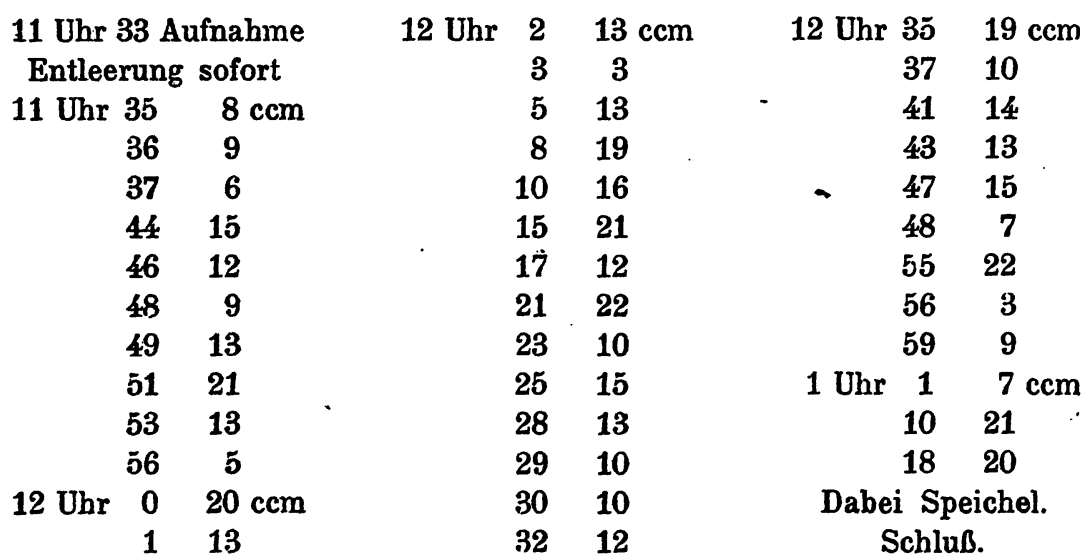

Im ganzen aufgefangen $493 \mathrm{ccm}$, also $243 \mathrm{ccm}$ Sekret. Dauer 115 Minuten.

1) O. Cohnheim u. P. Klee, Diese Zeitschrift, Bd. 78, S. 464, 1912. 
Ein zweiter Versuch wurde in ganz gleicher Weise an demselben Hunde ausgeführt. Die Dauer betrug 118 Minuten, die Sekretmenge (Magen- und Pankreassaft) $240 \mathrm{ccm}$.

Ein weiterer Versuch mit $20 \mathrm{~g}$ Hapan an einem Hunde mit einer gewöhnlichen Duodenalfistel ergab eine Sekretmenge von $330 \mathrm{ccm}$ (Magen-, Pankreassaft und Galle).

In einem weiteren Versuche wurden einem Hunde mit Magenfistel $300 \mathrm{ccm}$ Wasser mit $20 \mathrm{~g}$ Hapan direkt in die Magenfistel eingeführt und das Entleerte aus der Duodenalfistel aufgefangen und in sie eingespritzt. Dadurch sollte die psychische Sekretion nach Möglichkeit ausgeschaltet werden, doch ist es fraglich, ob das völlig erreicht wurde. Die Entleerung begann auch hier sofort und erfolgte so rasch, daß ich mit der Einspritzung kaum nachkommen konnte. In 6 Minuten wurden $153 \mathrm{ccm}$ entleert. Dann aber verlangsamte sich die Entleerung, die Gesamtdauer betrug 101 Minuten, die Sekretmenge (Magen-, Pankreassaft, Galle) $240 \mathrm{ccm}$. Durch den Wegfall oder die Verringerung der psyjchischen Sekretion waren Entleerungsdauer und Sekretmenge vermindert, blieben aber immer noch hoch. - 2 Versuche mit Erepton ergaben ähnliche Werte.

Aus diesen Versuchen ergibt sich, daß die abgebaute Nahrung sich in Magen und Dünndarm nicht anders verhält, wie die Nahrungsmittel, aus denen sie entstanden ist. Die chemische Einwirkung, die wohl in der Hauptsache von den Extraktivstoffen des Fleisches herrührt, hat genügt, um die fehlende Konsistenz zu ersetzen. Hapan enthält 10\% Stickstoff, $20 \mathrm{~g},{ }^{1}$ ) also $2 \mathrm{~g}$ Stickstoff, das entspricht dem Stickstoffgehalt nach etwa $60 \mathrm{~g}$ Fleisch. Nach Best ${ }^{1}$ ) werden auf $200 \mathrm{~g}$ Fleisch $1179 \mathrm{ccm}$ von allen Sekreten abgesondert, das würden auf $60 \mathrm{~g} 350 \mathrm{ccm}$ sein. Diese Menge ist nahezu erreicht. Fällt die Galle weg, so werden nach den Zahlen von Cohnheim und Klee auf $60 \mathrm{~g}$ Fleisch $300 \mathrm{ccm}$ abgesondert. Die Zahlen für die abgebaute Nahrung bleiben hinter diesen Zahlen nur ganz unbedeutend zurück. Die Ereptonzahlen verhalten sich nicht anders.

1) F. Best, Deutsches Arch. f. klin. Med., Bd. 104, S. 94, 1911. 
Der Organismus ergießt also auf die abgebaute Nahrung, die schon verdaut ist, ebensoviel oder fast ebensoviel Sekret, wie auf die unveränderte Nahrung, und die völlig gelöste abgebaute Nahrung passiert den Magen auch nicht schneller wie die ursprünglichen Nahrungsmittel. Das letztere gilt natürlich nur bei erhaltenem Pylorusreflex. Ich ließ 2 Hunde je $20 \mathrm{~g}$ Hapan in $300 \mathrm{ccm}$ Wasser saufen, spritzte aber das aus der Duodenalfistel ablaufende nicht ein. In den beiden Versuchen wurden 100 bezw. $127 \mathrm{ccm}$ Magensaft abgesondert, auf Grund des Wohlgeschmacks und der chemischen Wirkung der Extrativstoffe. Aber der Magen entleerte sich in 20-25 Minuten. Ich habe früher gezeigt, daß auf den Pylorus 2 Reflexe wirken, ein mechanischer vom Mageninneren und ein chemischer vom Dünndarm her. Nur der letztere kann bei der flüssigen Nahrung erhalten sein, die abgebaute Nahrung verhält sich hier wie natürliche flüssige Nahrung, etwa wie Milch. ${ }^{1}$ ) Sind aber die Reflexe erbalten, so wirkt die abgebaute Nahrung wie die unveränderte natürliche Nahrung.

Weiterhin habe ich nüchternen Hunden mit Duodenalund Magenfistel Lösungen von Hapan und Erepton und ebenso ein selbst hergestelltes Präparat von ganz abgebautem Fleisch ins Rectum eingeführt und dabei die Duodenal- bezw. Magenkanüle offen gelassen. Ich wollte sehen, ob die abgebaute Nahrung, wenn sie vom Dickdarm, vielleicht auch vom unteren Dünndarm, resorbiert würde, Sekretion von Verdauungssäften bewirkte. Dies war indessen nicht der Fall. Abderhalden, Frank und Schittenhelm haben angegeben, daß Erepton, das per clysma gegeben wird, glät resorbiert wird. Ich kann das für Erepton und ebenso für das andere Präparat, das Hapan, bestätigen, von dem ich bis zu $30 \mathrm{~g}$, in $200 \mathrm{ccm}$ Wasser gelöst, einführte, ohne daß es zu Stuhlgang kam. Aus den Kanülen aber tropften in einer Stunde nur einige Tropfen ab, nicht mehr als bei den gleichen Hunden auch auslief, wenn sie so, ohne Einführung, mit offenen Kanülen standen. Von den unteren Darmteilen her existiert also auch bei reichlicher Resorption

1) L. Tobler, Verhandl. d. deutsch. Gesellsch. f. Kinderheilkunde, Bd. 23. 
0. Cohnheim, Uber die Wirkung auf den Verdauungskanal.

keine Einwirkung der Abbauprodukte des Eiweißes oder der Extraktivstoffe auf die Drüsen. Daß auf diese Weise Eiweiß in normaler Weise resorbiert werden kann, ohne daß es dabei zu einer Tätigkeit der Verdauungsdrüsen kommt, kann zu einem prinzipiell wichtigen Schluß in bezug auf den Eiweißstoffwechsel verwendet werden. Grafe und Schöpp ${ }^{1}$ ) haben beobachtet, daß bei Einführung derselben zwei Präparate, die ich benutzt habe, in das Rectum die Stoffwechselsteigerung (spezifisch dynamische Wirkung) ebenso hoch ist, wie wenn eiweißhaltige Nahrung per os gegeben wurde. Sie schließen mit Recht daraus, daß damit die alte Frage nach der Ursache der spezifischdynamischen Wirkung des Eiweißes eindeutig in dem Sinne entschieden ist, daß sie nicht auf einer Tätigkeit der Verdauungsdrüsen beruhen kann. Durch meine Beobachtungen wird ein etwaiger Einwand gegen ihre Schlußfolgerung beseitigt.

1) P. Schöpp, Deutsch. Arch. f. klin. Med., 1913. Die Arbeit wurde mir durch die Liebenswürdigkeit von Herrn Dr. Grafe vor der Drucklegung bekannt. 